\section{Eye drop instillation technique in patients with glaucoma}

\begin{abstract}
Aims To evaluate the technique of eye drop instillation in patients with glaucoma and assess factors associated with a good technique. Methods A cross-sectional observational study of 85 participants using selfadministered topical medication for glaucoma or ocular hypertension. Patients were asked to demonstrate how they normally instil eye drops using a 5-ml bottle of sterile artificial tear solution. The procedure was recorded and assessed by two masked graders. Whether the patient had been previously shown how to instil drops, the number of eye medications used, and self-perceived difficulty of using drops were also recorded. Univariable logistic regression was performed to relate each variable to drop technique and variables with $P<0.2$ were included in a multivariable logistic regression analysis.

Results $54.1 \%(46 / 85)$ of patients had a poor drop technique, $11.8 \%(10 / 85)$ missed the eye, $15.3 \%(13 / 85)$ touched the tip of the bottle to the bulbar conjunctiva or cornea, and $27.1 \%$ (23/85) touched the eyelid or lashes with the bottle tip. $81.2 \%(69 / 85)$ could not recall being shown how to instil eye drops. In the multivariable model, previous instruction regarding drop instillation technique was significantly associated with good technique (adjusted OR $=8.17,95 \% \mathrm{CI}$ 2.02-33.05, $P=0.003$ ) and increasing age was associated with poor technique (adjusted $\mathrm{OR}=0.95,95 \%$ CI $0.91-0.99, P=0.017$ ).

Conclusions Education relating to eye drop instillation technique is significantly associated with a patient's ability to instil drops correctly. The assessment of a patient's ability to instil eye drops correctly should be a routine part of the glaucoma examination.

Eye (2013) 27, 1293-1298; doi:10.1038/eye.2013.187; published online 23 August 2013
\end{abstract}

AJ Tatham, U Sarodia, F Gatrad and A Awan

Keywords: glaucoma; compliance;

drop instillation; medical treatment; eye drop; education

\section{Introduction}

The successful medical treatment of glaucoma relies on adherence to a topical medication regimen. For some patients this includes multiple medications instilled several times per day. Previous studies have shown that patients commonly miss eye drop doses ${ }^{1-6}$ and that poor adherence is associated with more severe visual field loss. ${ }^{7}$ Kass et al, ${ }^{1}$ using an electronic dose monitor, found that $62 \%$ of patients omitted $10 \%$ of doses and $15 \%$ of patients omitted $50 \%$ of doses. Missed doses are often due to problems with medication adherence; however, even a patient with good compliance may not be receiving a correct dose because of problems with the technique of drop administration.

Approximately $80 \%$ of patients instil their own eye drops ${ }^{8}$ and there are many techniques used. ${ }^{9,10}$ Some patients instil their drops when sitting, others stand or lie down, others use a mirror to aid the process. ${ }^{11}$ If eye drop instillation is done improperly it can lead to treatment failure, unnecessary use of additional medications, and potentially to the spread of infection. ${ }^{7,12-14}$ Poor techniques can include missing the eye completely, delivery of an excessive dose, or ocular trauma or bottle contamination due to contact between the tip of the bottle and the globe or lid. Moreover, if the patient has a poor technique they are often unaware of the problem. ${ }^{13}$

Previous studies have identified several factors associated with an increased risk of poor eye drop instillation technique. For example, a poor technique is more likely in those with poor manual dexterity, poor vision, limited schooling, and older age. 1,15,16 There is,
Department of Ophthalmology, University Hospital Leicester, Leicester, Leicestershire, UK

Correspondence:

Department of Ophthalmology, University Hospital Leicester, Infirmary Square, Leicester, Leicestershire LE1 5WW, UK Tel: +44 (0)300303 1573; Fax: +44 (0)11 6258 5631; E-mail: andrewtatham@ yahoo.co.uk

Received: 9 January 2013 Accepted in revised form: 15 June 2013 Published online: 23 August 2013 
however, little evidence as to whether education regarding how to self-administer eye drops is associated with a better eye drop instillation technique. Lack of education is likely to be a large problem, as when clinicians prescribe eye drops, proper explanation and demonstration of how the eye drops should be used is often neglected. ${ }^{11}$ The aim of the present study was to investigate the technique of eye drop instillation in patients with glaucoma and assess the factors associated with good technique. Specifically, we aimed to evaluate whether previous education regarding eye drop administration was associated with better technique.

\section{Materials and methods}

This was a cross-sectional observation study of 85 subjects attending the glaucoma clinic at a university hospital.

\section{Statement of ethics}

All applicable institutional and governmental regulations concerning the ethical use of human volunteers were followed during this research. All subjects gave informed consent before enrolment and the protocol followed the tenants of the Declaration of Helsinki. The local Institutional Review Board approved the study.

Consecutive patients with a diagnosis of glaucoma or ocular hypertension who were using one or more topical ocular hypotensive medications in one or both eyes were included. Subjects were excluded if they did not instil their own medications or had been using eye drops for less than 6 months. We did not include patients who were using compliance aids to deliver their eye drops. Three subjects declined to participate in the study, as they did not wish to be video recorded. Demographic data collected included age, race, gender, and duration of use of topical ocular hypotensives. Participants were asked to complete a questionnaire detailing factors such as level of education, how many different eye medications they were using, and if they were using multiple medications, how long they wait between each drop. They were also asked to grade how difficult they felt it to instil their drops using a scale from 1 to 10, with 1 being very easy to 10 being extremely difficult. Participants were also asked whether they recalled having had any previous instruction on how to instil eye drops, and if so, from whom.

Patients were then asked to demonstrate how they normally instil eye drops using a $5 \mathrm{ml}$ low-density polyethylene bottle of sterile artificial tear solution. Facilities were provided for the patient to wash their hands, use a mirror, to sit or to lie down depending on the patient's preference. The procedure was recorded using a digital video recorder. Patients who used drops in only one eye were asked to demonstrate drop instillation into this eye. Patients who used drops in both eyes were randomly assigned to demonstrate the technique in one eye only. If patients were unhappy with their first attempt they were permitted a second attempt but research staff gave no prompting.

The eye drop instillation was graded from the digital video recording by two graders masked to the demographic and clinical details of the patients. The graders were required to assign a score to each drop instillation sequence ranging from -1 to 4 as detailed in Table 1 . The score was devised so that a score of 4 indicated the best technique and a score of -1 the worst technique. For the purposes of the scoring system, a patient who missed the eye was awarded a lower score than a patient who delivered the drop to the eye or conjunctival sac but touched the eyelid or globe with the tip of the bottle. The patient was deemed to have a good technique if the video score was 3 or 4 , and to have a poor technique if the video score was -1 to 2 . When there was disagreement between graders, a consensus grading was used.

\section{Statistical analysis}

Normality assumption was assessed by inspection of histograms and using Shapiro-Wilk tests. Descriptive statistics included $t$-test for normally distributed variables and Wilcoxon rank-sum test for continuous

Table 1 Scheme used to grade videos of eye drop instillation technique

\begin{tabular}{lr}
\hline Description of technique & Score \\
\hline Good technique, is on target, delivers a drop to the eye, and does not contaminate the bottle & 4 \\
Awkward technique, but is on target, delivers a drop to the eye, and does not contaminate the bottle & 3 \\
On target and delivers a drop to the eye but contaminates the bottle by touching the bottle tip to the lashes or lid & 2 \\
On target and delivers a drop to the eye but contaminates the bottle by touching the bottle tip to bulbar conjunctiva or cornea & 1 \\
(lower score due to added risk of trauma to ocular surface) & 0 \\
Not on target and misses the eye with the eye drop & -1 \\
Patient misses the eye with the eye drop and contaminates the bottle tip by touching the eye, eyelid or lashes & \\
\hline
\end{tabular}


non-normal variables. Fishers exact test was used for categorical variables. Univariable logistic regression was performed to relate each variable to drop technique (good technique defined as a score of 3 or 4 versus bad technique defined as a score of -1 to 2 ). A multivariable logistic regression analysis was used to assess those variables with univariable significance of 0.2 or less. Variables studied included gender, age, race, education, number of glaucoma medications, number of years of eye drop use, mean deviation in better eye, mean deviation in worse eye, visual acuity in better eye, and visual acuity in worse eye.

The robustness of the drop instillation technique scoring system was assessed using the Kappa coefficient of agreement among graders. ${ }^{17}$ The Kappa coefficient adjusts the observed proportional agreement to take into account agreement that would be expected by chance, with Kappa $=1$ indicating perfect agreement and Kappa $=0$ no agreement. ${ }^{18}$ Confidence intervals for Kappa were obtained using 1000 bootstrap samples. All statistical analyses were performed with commercially available software (Stata, version 12; StataCorp LP, College Station, TX, USA). The $\alpha$-level (type 1 error) was set at 0.05 .

\section{Results}

There were a total of 85 patients recruited including 74 Caucasians, 10 South Asians, and 1 African-Caribbean. There were 46 male and 39 female patients. Table 2 shows the demographic and clinical characteristics of the included patients.

The mean age of patients was $71.6 \pm 11.6$ years. Forty-six of 85 patients $(54.1 \%$ ) had a poor drop technique identified (score of -1 to 2). Sixty-nine of 85 (81.2\%) patients could not recall ever being shown how to instil their drops. Only 14 of 85 (16.5\%) recalled having been shown how to use their drops by an ophthalmic professional. Of patients with a poor technique, 10 $(10 / 85,11.8 \%)$ missed the eye with the drop (score of 0 ), $13(13 / 85,15.3 \%)$ touched the tip of the bottle to the bulbar conjunctiva or cornea, and $23(23 / 85,27.1 \%)$ touched the eyelid or lashes with the tip of the bottle. Twenty-five subjects $(25 / 85,29.4 \%)$ had the best technique score of 4 , 14 subjects $(14 / 85,16.5 \%)$ had a score of 3 . There were no subjects with a score of -1 . The mean ( \pm standard deviation) score assigned by the graders for drop technique was $2.4 \pm 1.4$. There was excellent agreement in drop technique score assigned by the graders (Kappa $=0.925,95 \%$ CI 0.863-0.985; $P<0.001)$.

The mean number of drops used was $1.7 \pm 0.8$. Fourteen of 85 subjects $(16.5 \%)$ were using three medications and 33 of 85 subjects $(38.8 \%)$ were using two medications. Nine of 50 subjects $(18 \%)$ using more than
Table 2 Demographic and clinical characteristics

\begin{tabular}{|c|c|}
\hline & Number of subjects (\%) \\
\hline \multicolumn{2}{|l|}{ Age (years) } \\
\hline$<50$ & $4(4.7)$ \\
\hline $50-69$ & $35(41.2)$ \\
\hline $70-89$ & $42(49.4)$ \\
\hline$\geq 90$ & $4(4.7)$ \\
\hline \multicolumn{2}{|l|}{ Gender } \\
\hline Male & $46(54.1)$ \\
\hline Female & $39(45.9)$ \\
\hline \multicolumn{2}{|l|}{ Ethnicity } \\
\hline Caucasian & $74(87.1)$ \\
\hline Afro-Caribbean & $1(1.2)$ \\
\hline South Asian & $10(11.8)$ \\
\hline \multicolumn{2}{|l|}{ Level of education } \\
\hline Basic schooling & $52(61.2)$ \\
\hline GCSE equivalent (age 16) & $13(15.3)$ \\
\hline A-level equivalent (age 18) & $12(14.1)$ \\
\hline University & $8(9.4)$ \\
\hline \multicolumn{2}{|l|}{ Mean deviation worse eye (dB) } \\
\hline$\leq 5$ & $31(36.5)$ \\
\hline $5-15$ & $24(28.2)$ \\
\hline$>15$ & $30(35.3)$ \\
\hline \multicolumn{2}{|l|}{ Duration of eye drop use } \\
\hline$<1$ year & $3(3.5)$ \\
\hline $1-5$ years & $29(34.1)$ \\
\hline $5-10$ years & $16(18.8)$ \\
\hline$>10$ years & $37(43.5)$ \\
\hline \multicolumn{2}{|l|}{ Previous drop education } \\
\hline No & $69(81.2)$ \\
\hline Yes- family member & $2(2.4)$ \\
\hline Yes- professional & $14(16.5)$ \\
\hline
\end{tabular}

one drop reported that they ordinarily instil the second drop immediately after the first, without any interval. Patients had been using anti-glaucoma eye drops for a mean of $9.8 \pm 7.8$ years (range $0.6-32$ years). Those with a good technique (score of 3 or 4 ) had been using eye drops for mean of $8.2 \pm 1.3$ years compared with those with a poor technique (score of -1 to 2 ) who had been using drops for a mean of $11.1 \pm 7.4$ years. There was no significant difference in the duration of drop use between those with a good or poor technique $(P=0.087)$.

On average, patients thought drop instillation was not difficult and assigned a mean drop instillation difficulty score of $2.6 \pm 1.9$. Thirty-four of 85 patients $(40 \%)$ graded difficulty as 1 out of 10,18 of 85 patients (21.2\%) graded difficulty as 2 out of 10 , and 13 of 85 patients (15.3\%) as 3 out of 10 . Only nine patients thought instilling their drops deserved a difficulty score of more than 5 out of 10 . There was no difference in the patient perception of drop difficulty between those with a good and those with a 
poor technique $(P=0.429)$. There was also no significant difference in gender $(P=0.282)$, ethnicity $(P=1.000)$, education level $(P=0.824)$, visual acuity in the better $(P=0.073)$ or worse eye $(P=0.820)$, mean deviation in the better $(P=0.373)$ or worse eye $(P=0.645)$, duration of eye drop use $(P=0.087)$, number of eye drops used $(P=0.087)$, and self-perceived difficulty of drop instillation $(P=0.429)$ between those with a good and poor technique (Table 3 ).

The univariable analysis showed younger age and a history of previous education regarding eye drop instillation technique were the only factors significantly associated with a good technique $(P<0.05$ for both). Table 4 shows the results of the univariable logistic regression for predicting good drop instillation technique. Variables with a $P<0.2$ in the univariable analysis were included in the multivariable regression model.

In the multivariable model, only age and previous instructions on drop instillation technique remained significant. Older age was associated with decreased odds of having a good technique of drop instillation, when previous education was accounted for, with a 0.95 -fold reduction in odds (adjusted OR $=0.95,95 \% \mathrm{CI}$ $0.91-0.99, P=0.017)$. Previous education regarding drop technique was associated with 8.17-fold increased odds of a good drop technique, controlling for age (adjusted $\mathrm{OR}=8.17,95 \%$ CI 2.02-33.05, $P=0.003$ ).

\section{Discussion}

The results of this study indicate that difficulty with selfinstillation of eye drops is a significant problem for patients with glaucoma. Forty-six of 85 patients $(54.1 \%)$ had a poor drop technique. For most patients with poor technique, the problem was contact between the tip of the bottle and the globe or eyelid; however, 10 of 85 patients $(11.8 \%)$ received no medication, as their attempt to deliver the drop missed the eye completely. If a patient was to consistently fail to successfully instil their eye

Table 3 Descriptive statistics comparing patients with a good eye drop instillation technique to those with a poor technique

\begin{tabular}{lccc}
\hline & Good technique $(n=39$ subjects) & Poor technique $(n=46$ subjects) & P value \\
\hline Age (years) & $68.6 \pm 12.4$ & $74.2 \pm 10.3$ & $0.028^{\mathrm{a}}$ \\
Gender & $20(51 \%)$ Female & $19(41 \%)$ Female & $0.282^{\mathrm{b}}$ \\
Ethnicity & $34(87 \%)$ Caucasian & $40(87 \%)$ Caucasian & $1.000^{\mathrm{b}}$ \\
Level of education & $23(59 \%)$ Basic & $29(63 \%)$ Basic & $0.824^{\mathrm{b}}$ \\
& $16(41 \%)$ Higher & $17(37 \%)$ Higher & $0.10 \pm 0.14$ \\
VA better eye (logMAR) & $0.05 \pm 0.11$ & $0.27 \pm 0.25$ & $0.073^{\mathrm{a}}$ \\
VA worse eye (logMAR) & $0.28 \pm 0.28$ & $-3.6 \pm 4.9$ & $0.820^{\mathrm{a}}$ \\
MD better eye (dB) & $-3.8 \pm 3.5$ & $-10.3 \pm 8.3$ & $0.373^{\mathrm{a}}$ \\
MD worse eye (dB) & $-10.7 \pm 7.3$ & $4(9 \%)$ Yes & $0.645^{\mathrm{a}}$ \\
Previous drop education & $12(31 \%)$ Yes & $42(91 \%)$ No & $2.012^{\mathrm{b}}$ \\
Self-perceived difficulty (1 = very easy, $10=$ very difficult) & $27(69 \%)$ No & $1.8 \pm 0.9$ & $0.429^{\mathrm{a}}$ \\
Number of eye drops & $2.4 \pm 1.8$ & $11.1 \pm 7.4$ & $0.087^{\mathrm{a}}$ \\
Duration of eye drop use (years) & $1.6 \pm 0.9$ & $0.087^{\mathrm{a}}$ \\
\hline
\end{tabular}

Abbreviations: VA, visual acuity; MD, mean deviation in decibels (dB).

a $t$-test.

${ }^{\mathrm{b}}$ Fisher's exact test.

Table 4 Results of univariable logistic regression for predicting good drop instillation technique

\begin{tabular}{|c|c|c|c|}
\hline & Odds ratio & $95 \% C I$ & $\mathrm{P}$ value \\
\hline Age (years) & 0.96 & $0.92-1.00$ & 0.026 \\
\hline Gender $($ male $=0)$ & 1.64 & $0.69-3.88$ & 0.261 \\
\hline Ethnicity $($ Caucasian $=0)$ & 0.71 & $0.20-2.53$ & 0.594 \\
\hline Level of education (basic schooling $=0$, higher schooling $=1$ ) & 1.19 & $0.56-3.21$ & 0.701 \\
\hline VA in better eye (logMAR) & 0.04 & $0.00-1.46$ & 0.066 \\
\hline VA in worse eye (logMAR) & 1.21 & $0.24-6.20$ & 0.818 \\
\hline MD in better eye $(\mathrm{dB})$ & 1.00 & $0.92-1.10$ & 0.917 \\
\hline MD in worse eye $(\mathrm{dB})$ & 0.99 & $0.94-1.04$ & 0.704 \\
\hline Previous drop education & 6.74 & $1.76-25.9$ & 0.002 \\
\hline Self-perceived difficulty $(1=$ very easy, $10=$ extremely difficulty $)$ & 0.91 & $0.73-1.14$ & 0.421 \\
\hline Number of eye drops & 0.58 & $0.32-1.06$ & 0.068 \\
\hline Duration of eye drop use (years) & 0.95 & $0.90-1.00$ & 0.075 \\
\hline
\end{tabular}

Abbreviations: VA, visual acuity; MD, mean deviation in decibels (dB). 
drops, medical treatment would be unsuccessful and there would be a higher risk of disease progression. There would also be a risk that the clinician introduces additional drops that would also be ineffective and could lead to local or systemic side effects.

Twenty-six of 85 patients (30.5\%) touched the tip of the bottle to eye, eyelid, or lashes. Touching the eye and lid with the tip of the bottle is a problem due to possible bottle contamination. ${ }^{12,19}$ Contamination of eye drops has the potential to cause ocular surface irritation, conjunctival inflammation, and discontinuation of medication, and, in eyes that have had previous glaucoma surgery, contamination may lead to blebrelated infections. Although the risk of contamination is likely to be higher in non-preserved preparations, contamination of preserved anti-glaucoma medications can also occur. Indeed, the rate of contamination of topical anti-glaucoma medications containing benzalkonium chloride has been reported to be as high as $28 \% .^{12}$ The most common microorganisms cultured in glaucoma eye drops are commensal skin and conjunctival flora such as the Gram-positive organisms Staphylococcus epidermidis and Staphylococcus aureus. ${ }^{12}$ Although microbial contamination may go unnoticed, there is the potential for serious complications, such as bacterial keratitis, especially if there is coexistent ocular surface disease. ${ }^{20}$

Previous studies have also found high rates of poor drop technique. For example, Brown et al ${ }^{13}$ reported that $13 \%$ of patients were unable to place a drop in their eye after one attempt and $80 \%$ touched the bottle tip to the lid or lashes. In other studies, the incidence of missing the eye when attempting to instil an eye drop has ranged from 6.8 to almost $20 \%$, and the incidence of contaminating the bottle tip has ranged from 20 to over $70 \% .{ }^{14,21}$ Factors associated with poor technique have also been examined with older age, ${ }^{15}$ limited school education, ${ }^{15,16}$ and poor vision ${ }^{16}$ reported to be of importance. Brown et al ${ }^{13}$ found that patients attending private practice were more likely to have a good eye drop instillation technique and suggested that this was because the private patients received better instructions on the use of drops; however, the effect of education on instillation technique was not directly examined. ${ }^{13}$ The role of previous education regarding eye drop instillation on the patient's ability to instil eye drops was investigated in the present study. We found a history of previous eye drop education was strongly associated with good instillation technique, with 8.17-fold increased odds of a good technique, controlling for age. Older age was also a significant factor-associated drop technique with 0.95 lower odds of a good technique for each year of advancing age. Factors such as level of schooling, visual acuity, mean deviation, and self-perceived difficulty of drop instillation, were not significantly related to the ability to correctly instil eye drops.

The results of this study indicate that more should be done to educate patients regarding correct drop instillation technique, particularly as more than $80 \%$ of patients had never received, or could not recall receiving, any instructions on how best to instil their eye drops. Moreover, patients with a poor technique were mostly unaware that they had a problem with drop instillation. Over $60 \%$ of those with a poor technique graded the difficulty of drop instillation as 1 or 2 out of 10, where 1 denotes very easy and 10 very difficult. Patients with glaucoma should be taught a good technique of instilling eye drops, and we would suggest that this is done when the drop is first prescribed. The patient should then be observed performing the correct technique and if the patient has problems with the technique, a compliance aid could be tried. Reinforcement of the correct technique should then occur at future visits, with periodic observation of the patient instilling his or her own eye drops. This approach to education is also likely to help motivate the patient to adhere to the medication regimen.

Patient education requires time and financial resources, which are increasingly scarce in the healthcare setting; however, investment in patient education is likely to be cost effective and improve patient satisfaction. Failure to deliver the desired drug to the eye leads to wasted medication and poor intraocular pressure control, and is likely to lead to frequent changes in medication and more frequent hospital episodes. Patients who fail to respond to medical treatment require significant resources and are at increased risk of treatment escalation, including surgery. In contrast, good drop instillation technique has the potential to improve drug delivery, increase the effectiveness of treatment, and therefore potentially reduce the number of patient visits. In other words, if resources are deployed to improve patients' drop technique, there are likely to be savings elsewhere.

The study design has limitations. For example, the history of previous education regarding drop instillation technique relied on patient recall. It is possible that patients with poor drop instillation technique may have forgotten previous instruction regarding eye drop instillation, biasing the results. Furthermore, a portion of the data was collected using a questionnaire unique to this study, and therefore not previously validated. Bias may also have been introduced due to the setting of the study in the hospital glaucoma clinic. Patients may have felt being under pressure to perform as they were observed and filmed by a medical professional. This may have had a negative or positive impact on performance. Also, we did not assess whether the patient was left or 
right handed, which may have influenced the ability to instil drops, as the eye tested was randomly assigned. A further limitation of this study is that we did not examine the role of compliance aids, which can improve drop instillation for some patients and have been reported to significantly reduce touching of the bottle tip to the eye and eyelid. ${ }^{22}$ We decided not to include compliance aids in this study, as they are often suitable for only a single bottle design and many patients find it easier to use drops without such a device. ${ }^{23}$

Despite the limitations, this study has shown that previous education regarding eye drop instillation technique is significantly associated with a patient's ability to instil drops correctly. We recommend that drop instillation technique is routinely taught and assessed for patients with glaucoma.

\section{Summary}

What was known before

- Difficulty with self-instillation of eye drops is a problem for many patients with glaucoma.

- Improper drop instillation can lead to treatment failure and unnecessary use of additional medications.

- Reasons for poor technique include poor vision, arthritis, and old age.

\section{What this study adds}

- Many patients have never received instruction on how best to instil their drops.

- Most patients with a poor drop instillation technique are unaware they have a problem.

- The strongest factor associated with a poor eye drop instillation technique was lack of prior education regarding the correct use of eye drops.

\section{Conflict of interest}

The authors declare no conflict of interest.

\section{References}

1 Kass MA, Meltzer DW, Gordon M, Cooper D, Goldberg J. Compliance with topical pilocarpine treatment. Am J Ophthalmol 1986; 101: 515-523.

2 Winfield AJ, Jessiman D, Williams A, Esakowitz L. A study of the causes of non-compliance by patients prescribed eyedrops. Br J Ophthalmol 1990; 74: 477-480.

3 Granström PA. Glaucoma patients not compliant with their drug therapy: clinical and behavioural aspects. Br J Ophthalmol 1982; 66: 464-470.

4 Lu VH, Goldberg I, Lu CY. Use of glaucoma medications: state of the science and directions for observational research. Am J Ophthalmol 2010; 150: 569-574.
5 Stryker JE, Beck AD, Primo SA, Echt KV, Bundy L, Pretorius GC et al. An exploratory study of factors influencing glaucoma treatment adherence. J Glaucoma 2010; 19: 66-72.

6 Tsai JC, McClure CA, Ramos SE, Schlundt DG, Pichert JW. Compliance barriers in glaucoma: a systematic classification. I Glaucoma. 2003; 12: 393-398.

7 Sleath B, Blalock S, Covert D, Stone JL, Skinner AC, Muir K et al. The relationship between glaucoma medication adherence, eye drop technique, and visual field defect severity. Ophthalmology 2011; 118: 2398-2402.

8 Kass MA, Hodapp E, Gordon M, Kolker AE, Goldberg I. Part I. Patient administration of eyedrops: interview. Ann Ophthalmol 1982; 14: 775-779.

9 Fraunfelder FT. Extraocular fluid dynamics: how best to apply topical ocular medication. Trans Am Ophthalmol Soc 1976; 74: 457-487.

10 Zimmerman TJ, Kooner KS, Kandarakis AS, Ziegler LP. Improving the therapeutic index of topically applied ocular drugs. Arch Ophthalmol 1984; 102: 551-553.

11 Tsai T, Robin AL, Smith III, JP. An evaluation of how glaucoma patients use topical medications: a pilot study. Trans Am Ophthalmol Soc 2007; 105: 29-35.

12 Geyer O, Bottone EJ, Podos SM, Schumer RA, Asbell PA. Microbial contamination of medications used to treat glaucoma. Br J Ophthalmol 1995; 79: 376-379.

13 Brown MM, Brown GC, Spaeth GL. Improper topical self-administration of ocular medication among patients with glaucoma. Can J Ophthalmol 1984; 19: 2-5.

14 Gupta R, Patil B, Shah BM, Bali SJ, Mishra SK, Dada T. Evaluating eye drop instillation technique in glaucoma patients. J Glaucoma 2012; 21: 189-192.

15 Kholdebarin R, Campbell RJ, Jin YP, Buys YM. Multicenter study of compliance and drop administration in glaucoma. Can J Ophthalmol 2008; 43: 454-461.

16 Dietlein TS, Jordan JF, Lüke C, Schild A, Dinslage S, Krieglstein GK. Self-application of single-use eyedrop containers in an elderly population: comparisons with standard eyedrop bottle and with younger patients. Acta Ophthalmol 2008; 86: 856-859.

17 Cohen J. Weighted kappa: nominal scale agreement provision for scaled disagreement or partial credit. Psychol Bull 1968; 70: 213-220.

18 Landis JR, Koch GG. The measurement of observer agreement for categorical data. Biometrics 1977; 33: 159-174.

19 Kim MS, Choi CY, Kim JM, Woo HY. Microbial contamination of multiply used preservative-free artificial tears packed in reclosable containers. Br J Ophthalmol 2008; 92: 1518-1521.

20 Schein OD, Wasson PJ, Boruchoff SA, Kenyon KR. Microbial keratitis associated with contaminated ocular medications. Am J Ophthalmol 1988; 15(105): 361-365.

21 Stone JL, Robin AL, Novack GD, Covert DW, Cagle GD. An objective evaluation of eyedrop instillation in patients with glaucoma. Arch Ophthalmol 2009; 127: 732-736.

22 Nordmann JP, Baudouin C, Bron A, Denis P, Rouland JF, Sellem E et al. Xal-Ease: impact of an ocular hypotensive delivery device on ease of eyedrop administration, patient compliance, and satisfaction. Eur J Ophthalmol 2009; 19: 949-956.

23 Salyani A, Birt C. Evaluation of an eye drop guide to aid self-administration by patients experienced with topical use of glaucoma medication. Can J Ophthalmol 2005; 40: 170-174. 